\title{
IMPACTO DE LAS ACTIVIDADES PRODUCTIVAS Y DE LA EXPANSIÓN URBANA SOBRE UNA RESERVA ECOLÓGICA; EL CASO DE CUXTAL, YUCATÁN; MÉXICO
}

\section{Impact of productive activities and urban expansion} on an ecological reserve; Cuxtal's case in Yucatán; Mexico

Rafael Ortiz-Pech ${ }^{1}$, Henry Emmanuel Celis Fajardo², Gerardo García Gil ${ }^{3}$

Fecha de recepción: 22 de febrero de 2016.

Fecha de aceptación: 11 de mayo de 2016.

1 - Nacionalidad: Mexicana. Grado: Doctorado en Economía. Especialización: Desarrollo regional, Economía rural. Cadenas de valor. Ordenamientos Ecológicos y territoriales. Contabilidad social y simulación dinámica. Adscripción: Universidad Autónoma de Yucatán.Correo electrónico: rafael.ortiz@correo.uady.mx; ortizpech@hotmail.com

2- Nacionalidad: Mexicana. Grado: Estudiante tesista de Licenciatura en Economía. Especialización: Economía. Adscripción: Universidad Autónoma de Yucatán. Correo electrónico: hcelis.fajardo@outlook.com

3- Nacionalidad: Mexicana. Grado: Doctorado en Geografía. Especialización: Cartografía, sistemas de información geográfica, evaluación de recursos naturales y usos de suelo. Adscripción: Universidad Autónoma de Yucatán. Correo: garciag@correo.uady.mx 


\section{Resumen}

Este artículo analiza las condiciones socioeconómicas entre los habitantes de la Reserva Ecológica Cuxtal (REC), la cual sufre los efectos de la expansión urbana sobre la cobertura forestal. Para conocer su situación actual se aplicaron 400 cuestionarios en comunidades que se localizan al interior de la REC. Se describen los aspectos socioeconómicos y actividades productivas que provocan afectaciones a la reserva, como el cambio de uso del suelo. Los resultados del trabajo indican que los habitantes de la reserva no tienen conocimientos básicos del área en protección. De igual modo se presenta un modelo PROBIT la cual analiza cómo la población es motivada a realizar trabajos fuera de la reserva a causa del bajo nivel de ingreso.

Palabras clave: Reserva ecológica, afectación humana, actividades productivas, población, movilidad laboral.

\section{Abstract}

This article analyzes the socioeconomic conditions between inhabitants of the Cuxtal Ecological Reserve (REC), which suffers the effects of urban sprawl on forest cover. It was applied randomly 400 surveys in several communities located within REC to meet socioeconomic aspects and describe some productive activities that cause damages to the reserve. The results of the study indicates that the inhabitants of the reserve have not basic knowledge of the protection area. Similarly a PROBIT model analyzes how people are motivated to perform work outside the reserve due to the low level of weekly income obtained.

Key words: Ecological reserve, human affectation, productive activities, population, labor mobility. 


\section{Introducción}

$\mathrm{E}$

n la actualidad se puede afirmar que el fenómeno de la expansión urbana está presente en todas las ciudades del mundo, sin embargo el proceso de expansión o crecimiento de las urbes resulta más contundente en las ciudades de los países emergentes. Las ciudades se expanden y en ocasiones se conurban dando lugar a zonas metropolitanas, como el caso de Mérida que se ha transformado en una ciudad policéntrica, rebasando límites políticos y administrativos. La principal causa del rápido crecimiento de las ciudades es debido a los movimientos migratorios generados por la población que las ciudades atraen, así como por su tasa de crecimiento natural. Las ciudades crecen aumentando inexorablemente sus periferias, devorando los espacios contiguos normalmente ocupados por las actividades del agro o del medio rural, dichos espacios sucumben a la presión de los inversionistas, de las compañías constructoras que logran urbanizar haciendo crecer lo urbano (Iracheta y Bolio, 2012).

En el caso del municipio de Mérida en donde se ubica la Reserva Ecológica Cuxtal ${ }^{4}$ (REC), la administración municipal desde el año de 1993 decretó la creación de un área de protección con el fin contener la expansión desordenada de la ciudad de Mérida, además garantizar un cinturón verde, que mantenga y mejore las condiciones ambientales al brindar los servicios ecológicos a la ciudad capital del estado de Yucatán. La acción de haber decretado un área para la conservación en los límites del área urbana se puede considerar como un intento de regular el crecimiento urbano y dotar a la ciudad de servicios ambientales con el fin de mejorar la calidad de vida de sus habitantes, sin embargo es evidente que dentro de un área natural protegida las actividades productivas deben ser restringidas a zonas de amortiguamiento de acuerdo con el plan de manejo de la Reserva, lo que puede ocasionar molestias o inconformidades entre los habitantes haciendo difícil la convivencia entre sociedad y reserva.

Si se consideran los problemas ambientales que enfrenta una sociedad en relación con el cambio climático y el calentamiento global se puede suponer la gran importancia de esta reserva del municipio de Mérida, no solo por ser la fuente de abastecimiento y extracción de agua para ser distribuida en la capital del estado, sino por la conservación y preservación de la riqueza en flora y fauna propia de las selvas bajas caducifolias del norte de la Península de Yucatán.

Partiendo de los supuestos de la planificación urbana y la conservación y respeto por la naturaleza, se puede afirmar que es conveniente crear Áreas Naturales Protegidas (ANP) sin soslayar que se deben dar alternativas productivas a los grupos humanos que viven en ellas, dichas actividades deben ser sustentables. Este trabajo muestra como el crecimiento de la población, y las actividades especulativas con el uso del suelo que se realizan dentro de la REC y como el trabajo realizado fuera de la reserva afecta a la conservación. Para realizar esta investigación, se plantean los antecedentes de la reserva, la justificación de este trabajo, la fundamentación teórica, la metodología aplicada, la descripción de la zona de estudio, los resultados así como las conclusiones y los anexos del trabajo.

4- La palabra "Cuxtal" es de origen maya yucateco (Kuxtal) y significa "vida o existencia" en español. 


\section{Antecedentes de la REC}

La REC fue promovida para su creación por el Ayuntamiento de Mérida ${ }^{5}$ como un área de protección y amortiguamiento para la ciudad por la administración del gobierno municipal del periodo 1991-1993 pero para el final del periodo (1993) se logró la aprobación de su establecimiento. Un objetivo de su creación fue conservar el manto freático de esa zona pues allí se extrae agua potable para ser distribuido a la ciudad de Mérida.

La REC está conformada dentro de comisarías del municipio de Mérida y han tenido características rurales y urbanas por lo que desde su establecimiento, la mancha urbana ha crecido y ha impactado a la reserva; pero que ningún gobierno ha podido controlar dicho crecimiento. De hecho, algunas administraciones gubernamentales han fomentado la construcción de fraccionamientos, vialidades y viviendas para trabajadores lo que provoca la introducción de servicios públicos como energía eléctrica, agua potable, pavimentación de calles, transporte público, etc; lo cual agrava la situación. También es importante mencionar que cada año entre finales de noviembre y principios de diciembre, la ciudad de Mérida festeja su feria ganadera y en febrero las fiestas de carnavales; ambas en la comisaría de Xmatkuil (comisaría dentro de la REC) lo que ha provocado la necesidad de un mayor espacio para el estacionamiento de los vehículos que se tradujo en la deforestación de la reserva ecológica.

Las afectaciones anteriores son muestra que la reserva ha sido deteriorada severamente en los últimos años no solamente por la falta de autoridad para preservarla sino también por la sociedad que la habita que no ha tenido el cuidado para mantenerla. La situación en la reserva se vuelve más crítica cuando algunos ejidatarios intentan vender sus propiedades ejidales a personas que buscan comprar tierras baratas para hacer negocios, agravando la estabilidad y las funciones de la reserva (García-Gil; Oliva-Peña y OrtizPech, 2012).

\section{Justificación del tema}

El programa de ordenamiento ecológico territorial como una propuesta para ordenar el uso del suelo pues el fomento de las actividades productivas en distintas áreas geográficas ha ocasionado conflictos ambientales (SEDUMA, 2016).

La zona metropolitana de la ciudad de Mérida se ha convertido en la mayor concentración poblacional de la Península de Yucatán y del sureste de México. Es necesario reflexionar sobre las relaciones y vínculos que deben construirse entre la REC y la población que habita el área así como los antagonismos que puedan surgir en esta relación. Esto es uno de los tópicos que este trabajo aborda, en la cual los pobladores que viven en el área enfrentan limitaciones para realizar cambios de uso del suelo, adicionalmente prefieren buscar trabajos en el área de servicios que son mejor remunerados y no dependen de las condiciones climatológicas que suelen afectar con frecuencia al cultivo de la milpa y otros productos agrícolas, así como de actividades pecuarias que se realizan en la zona (García-Gil y Escalante, J., 2013).

5- La Reserva Ecológica Cuxtal es una de las únicas reservas creadas baja una jurisdicción municipal en México y su creación fue promovida por la autoridad municipal según el Diario Oficial del gobierno del estado de Yucatán publicado el 4 de octubre de 2004 en la gaceta $\mathrm{N}^{\circ} 30227$ 
Por la importancia del tema se realizan proyecciones para la población que habita en la reserva y cómo podría afectar a la REC. De igual modo, se describen algunas actividades productivas y cotidianas que se realizan dentro y fuera de la reserva pero que afectan al sostenimiento de la reserva.

En general este trabajo toma como referencia que la ciudad de Mérida crece con relación a su población y la reserva es afectada en su parte norte. Únicamente para el año 2015, ya se afectó el $10 \%$ de la reserva y se estima que para 30 años más (2045) podría ser afectado el $20 \%$ de su área original, es decir el doble de lo actual. Esto implica una toma de decisión de política seria para mantener el "pulmón” de la ciudad capital aunque oficialmente ya se ha detectado el problema y se ha decretado en el Diario Oficial del Estado de Yucatán (2004) el documento rector que señala las estrategias y políticas de conservación las cuales deben ser aplicables dentro de la REC.

\section{Aspectos conceptuales y teóricos}

\subsection{ANP}

Las ANP'S son áreas creadas por las autoridades competentes como una opción para conservar flora y fauna de un área delimitada. Más específicamente, según Gonzáles; Cortés; Íñiguez y Ortega (2014) indican que de acuerdo al Convenio sobre la Diversidad Biológica, una ANP es "un área definida geográficamente que haya sido designado o regulado y administrado para alcanzar objetivos específicos de conservación".

Por su parte, la Comisión Nacional de Áreas Naturales Protegidas (CONANP) establece que "las Áreas Naturales Protegidas son las zonas del territorio nacional y aquellas sobre las que la nación ejerce su soberanía y jurisdicción en donde los ambientes originales no han sido significativamente alterados por la actividad del ser humano o que requieran ser preservadas y restauradas" CONANP (2016).

Ambas definiciones indican que cuando la biodiversidad de un área geográfica está en peligro de existencia se decretan estas áreas por la necesidad de conservarlos y protegerlos pues los recursos naturales existentes proveen beneficios ambientales benéficos para los que habitan la región (no solo humanos, sino flora y fauna silvestre). También pueden decretarse cuando la integridad de los ecosistemas sean amenazados por diversos factores tales como el aumento de la mancha urbana, cacería y extracción de flora ilegal, cambio en el uso del suelo, etc.

Por tanto, como una medida de contención del avance de la degradación, surgen las ANP cuyas funciones son diversas pero que buscan en primer lugar la conservación y que no afecten otras generaciones futuras; de modo que provean un beneficio para la sociedad con solo prestarle el cuidado y uso de sus recursos de manera sustentable y también al entorno natural en la cual se ubica (Ruiz, 2016).

Así, las ANP se establecen por decreto de gobierno y se rigen de acuerdo a la Ley General de Equilibrio Ecológico y Protección al Ambiente (LGEEPA), así como su reglamento y los programas de ordenamiento ecológico territorial correspondientes a su área de influencia. En conclusión, los sitios como ANP deberán ser lugares claves para la preservación de toda la riqueza en biodiversidad (SEDUMA, 2016). 


\subsection{El desarrollo Sustentable}

Debe existir una relación entre el sistema integrado por la economía, el ambiente y la sociedad dentro de una comunidad, país o región. Cuando no existe tal relación, estas entidades se consideran separadas o ausentes y por lo tanto surgen diversos problemas (Díaz y Escárcega, 2009).

Este equilibrio debe considerarse para la toma de decisiones políticas ya que cualquier intento de cambio en algún componente, provoca impactos en los otros integrantes del sistema y sus repercusiones en la región o comunidad son impactados. Esto invoca la necesidad de analizar los problemas de índole ambiental y económico dentro de un enfoque conocido como desarrollo sustentable. El desarrollo sustentable es considerado como un sistema en donde se privilegia el bienestar social, la habitabilidad, y la integridad del ambiente a fin de evitar externalidades negativas.

Sin embargo, uno de los problemas recurrentes en la actualidad es la falta de desarrollo sustentable (Miguel et al., 2011 y Merchant, 2011) y se reconoce su agravamiento. Cuando se refiere a la propiedad comunal de los recursos naturales, su administración puede complicarse cuando los recursos son usados de manera colectiva, tal como el suelo de tierras ejidales. En este caso, el enfoque sobre la tragedia de los comunes de Hardín (1968) puede ser planteado inicialmente para entender teóricamente la situación.

Hardín expuso el tema del famoso caso del pastizal en donde los pastores llevan a sus animales al mismo lugar a pastar. Debido a que el recurso que sirve de alimento es común, los pastores tratan de tener el mayor número de animales esperando tener mayor stock que puedan ser usados en otros tiempos (venta, autoconsumo, etc.), esto es por la conducta racional esperado del pastor que busca maximizar sus beneficios.

Sin embargo, este comportamiento puede tener dos puntos antagónicos, uno positivo y otro negativo. El positivo se refiere a que el pastor tiene una función creciente de ventas potenciales por el incremento de cabezas de sus animales (utilidad positiva), pero de igual modo, provoca un aspecto negativo relacionado con el deterioro del área de pastoreo debido al gran número de animales poseídos o sobrepastoreo.

El aspecto negativo ocurre debido a que existen otros pastores, que no se dan cuenta del problema o no valoran el agotamiento por lo que la utilidad de cada pastor es solo una fracción del total. Debido a esta apariencia de utilidad, los dueños de los rebaños tienden a añadir más animales a su rebaño provocando mayor agotamiento del pastizal o deterioro del suelo, lo que lleva a la tragedia de los comunes. Lo que parece bueno para uno, puede ser la ruina de todos, ya que cada pastor piensa en tener mayor beneficio; sin embargo, a nivel general, ante esta actitud llevan al agotamiento del recurso que sirve de insumo (pastizal) para la alimentación de los animales. Esta situación puede ser aplicada a otros casos, como en áreas de conservación que tienen una extensión física y recursos naturales limitados; pero la población puede acceder a los recursos de manera no limitada y afectar el área a costa de los usuarios o visitantes.

La explicación de Hardín ha provocado el surgimiento de otros enfoques teóricos para explicar la degradación de los recursos naturales comunales y sus posibles soluciones. Una de ellas es Ostrom (2000) que en su libro titulado "el gobierno de los bienes comunes" postula que la solución al problema de los bienes colectivos puede ser el establecimiento de políticas públicas que establezcan los derechos de propiedad sobre los recursos naturales o bien que el gobierno intervenga impositivamente para controlar tal degradación. Sin embargo, plantea una tercera opción; que los usuarios consideren la administración colectiva.

Es de notar, que Ostrom ofrece una diversidad de soluciones la cual por la intervención podría llevar al aumento de la eficiencia de modo que culmine de manera exitosa los resultados con la firma de un 
contrato en la que se compromete seguir una estrategia de cooperación. Sin embargo, el final del planteamiento no está garantizado ya que los ambientes son complejos, la sociedad puede fallar y las estrategias se pueden ejecutar o no (Rodríguez, 2010).

En conclusión, a pesar de esta nueva propuesta sobre el problema de la degradación de los bienes comunales, no se puede garantizar una solución de manera infalible ya que ni las leyes de mercado o de gobierno son suficientes, tampoco por la creación de organizaciones locales.

\section{Metodología}

Para poder cumplir los objetivos del trabajo se realizó un análisis de información y se utilizaron dos fuentes de datos primarios (directos) y secundarios (indirectos). Por las primeras, se aplicaron cuestionarios socioeconómicas muestrales a hogares que habitan la REC. Entre la información recabada directamente a los hogares se tienen aspectos socioeconómicos, percepción sobre la comunidad, características socioeconómicas de la población ocupada. Por las fuentes secundarias se usaron datos provenientes de páginas oficiales de estadísticas como del Instituto Nacional de Estadística y Geografía (INEGI), del Consejo Nacional de Población (CONAPO) y del Consejo Nacional para la Evaluación de la Política Social de Desarrollo Social (CONEVAL).

Con relación al trabajo primario, fue necesario aplicar cuestionarios socioeconómicos a los hogares que pertenecen a la REC visitando las nueve comunidades involucradas. La encuesta se aplicó durante el año 2014 y el análisis de ella se realizó durante el año 2015. El trabajo de campo fue financiado por el gobierno municipal de Mérida dentro del proyecto denominado "Programa de Ordenamiento Ecológico Territorial del Sur del municipio de Mérida" (POETSM).

La información obtenida fue capturada en una hoja de cálculo de Excel dividiendo los datos en tres grupos; información socioeconómica de los hogares, información del solar o traspatio y finalmente sobre la parcela. Además de lo anterior, se preguntó información sobre la percepción o conocimiento que tienen con respecto a la REC, valores culturales y naturales, comunicación entre autoridades de gobierno local con la población local para seis de las seis de nueve localidades de la REC.

Debido a la gran cantidad de población que habita en la REC, se obtuvo una muestra de la misma con la cual se realizó la encuesta socioeconómica. El tipo o técnica de muestreo utilizado fue el muestreo aleatorio simple para poblaciones finitas en la cual toda la población $(\mathrm{N})$ tiene la misma probabilidad de ser elegida como muestra (n) (Mendehall, Ott y Scheaffer, 1987).

El procedimiento para elegir los hogares por localidad fue enumerar las viviendas habitadas y seleccionarlos aleatoriamente teniendo en cuenta el tamaño de muestra mínimo que sea representativa. La estimación de la muestra se hizo aplicando la siguiente ecuación:

$$
n=\frac{N \cdot Z_{\propto}^{2} \cdot p \cdot q}{\left[(N-1) \cdot d^{2}\right]+\left[Z_{\propto}^{2} \cdot p \cdot q\right]}
$$


Donde:

$\begin{array}{ll}\mathrm{N}=\text { Total de la población } & \mathrm{n}=\text { Tamaño de muestra } \\ \mathrm{p}=\text { Proporción esperada } & \mathrm{Za}=\text { Distribución normal } \mathrm{Z} \\ \mathrm{q}=1-\mathrm{p} & \mathrm{d}=\text { Precisión }\end{array}$

De acuerdo al aplicar la ecuación anterior, el cuadro 1 muestra los resultados de las muestras necesarias para el trabajo de investigación. Es importante mencionar que el insumo previo a esto fue los croquis de las localidades y el conocimiento de las viviendas habitadas, datos que fueron obtenidos de INEGI (2010).

\section{Cuadro 1. Muestras por localidades en REC}

\begin{tabular}{|c|c|c|c|c|}
\hline Localidad & $\begin{array}{c}\text { Viviendas habitadas } \\
(\mathbf{n})\end{array}$ & $\mathbf{n}$ & $\begin{array}{c}\text { Nivel de confianza } \\
(\%)\end{array}$ & $\begin{array}{c}\text { Viviendas } \\
\text { entrevistadas }\end{array}$ \\
\hline Molas & 508 & 64 & 95 & 103 \\
\hline Dzununcán & 405 & 62 & 95 & 102 \\
\hline San Pedro Chimay & 277 & 58 & 95 & 79 \\
\hline Santa Cruz Palomeque & 192 & 53 & 95 & 75 \\
\hline Tahdzibichén & 164 & 51 & 95 & 66 \\
\hline Xmatkuil & 127 & 47 & 95 & 40 \\
\hline Dzoyaxché & 109 & 44 & 95 & 22 \\
\hline San Ignacio Tesip & 84 & 39 & 95 & 643 \\
\hline Hunxectamán & 38 & 15 & 90 & 51 \\
\hline Total & 1904 & & & 405 \\
\hline
\end{tabular}

Fuente: Celis (2015).

El error muestral admitido varió entre localidades, pero se puede visualizar que se ubicó entre el 5\% (8 casos) y el 10\% (un caso, Hunxectamán). Un asunto importante que se observa del cuadro 1 es la toma de un mayor tamaño muestral que la recomendada. El motivo para tal acción fue que se aplicó el supuesto que las viviendas habitadas eran mayor pues los datos considerados eran de INEGI (2010) y las encuestas se realizaron en 2014, es decir existía un desfase de cuatro años entre el censo de INEGI y la aplicación de los cuestionarios.

Una vez considerado lo anterior, se procedió a realizar las visitas a las viviendas para aplicar los cuestionarios socioeconómicos. El criterio de visita fue abarcar todos los puntos geográficos de las comunidades (norte, sur, oriente, poniente, centro, y otros puntos intermedios) de este modo que se garantice una buena distribución muestral. Toda la información recopilada se vació en hojas de cálculo de Excel para su posterior análisis estadístico y con STATA` (versión SE 14 ) para construir un modelo probit sencillo usando como variable dependiente "mov" y para la variable independiente "ysemanal" con el objetivo de mostrar cómo el movimiento laboral de la población ocupada de la REC se desplaza en dependencia con el ingreso semanal obtenido fuera de la reserva. 


\section{Zona de estudio}

La REC se encuentra en el municipio de Mérida dentro de la Península de Yucatán. El municipio tenía una población de 830,732 habitantes según INEGI (2010) y está conformado por 11 comisarías y 36 subcomisarias. La reserva se encuentra cerca de la principal ciudad urbana, Mérida, en la parte sur del municipio y aún se observa que las actividades agropecuarios son importantes aunque existen elementos que la economía campesina está en declive y la practican menos del 10\% de los hogares (Lugo y Tzuc, 2010).

Por tanto, estas localidades dentro de las REC presentan una característica muy particular pues tienen vínculos con lo urbano y rural de manera intensa. A estas localidades o comisarías se les conoce como rur urbanas o "nueva ruralidad" puesto que realizan actividades del sector primario aunque también desempeñan actividades propias de las zonas urbanas como actividades del sector secundario y terciario; inclusive se vinculan a un mercado laboral urbano que es donde obtienen los principales ingresos (Lugo y Tzuc, 2010).

Un contraste que existe en este municipio es la diferenciación socioeconómica de la población, pues mientras la principal zona urbana está clasificada como de muy baja marginación, las otras localidades que forman el municipio se encuentran con muy alto grado de marginación. Esto provoca la existencia de "dos Méridas", una en mejoras condiciones que la otra.

Esto no fue siempre así, pues desde los años 70's la ciudad crecía de manera ordenada ya que no había crecimiento poblacional acelerado para luego empezar a desarrollarse de manera desordenada entre los años de 1980 y 2000 cuando empezaron a especularse la compra de tierras ejidales, la construcción de centros comerciales, universidades, fraccionamientos, empresas e industrias, etc., provocando la expansión urbana y por tanto un cambio en el uso del suelo de la ciudad de Mérida.

Estos problemas con el uso del suelo se han dado de igual modo en la zona sur de Mérida pues se sabe de la venta de terrenos, aunque en menor escala que en otras zonas de la ciudad, el valor de estas tierras no tienen tanto valor que hasta los empresarios no tienen interés de adquirirlas por la poca plusvalía de la zona.

Las tierras que logran ser vendidas se destinan para fines de producción para el mercado de monocultivos tales como papaya maradol y otros cultivos perennes agropecuarios y en menor medida para viviendas habitacionales. Las subcomisarias que presentan mayor conurbación son Xmatkuil, Santa Cruz Palomeque y Tahdzibichén ya que se encuentran más cerca de la zona urbana de la ciudad de Mérida. En contraste, las localidades de Texán Cámara, San Pedro Chimay, Dzoyaxché, Yaxnic y Petac aún conservan mejor sus tierras debido a su lejanía o mayor aislamiento con Mérida. De hecho, en estas últimas comunidades aún es posible encontrar hogares que siembran maíz en sus milpas para destinarlas al autoconsumo y en algunos casos venden sus productos obtenidos producto del trabajo agropecuario. En ambos grupos de localidades es donde la REC se encuentra y se está en peligro.

Con respecto al área de la REC esta se creó en 1993 (Diario Oficial del Gobierno del Estado de Yucatán, 2004) con el fin de proteger el manto freático pues en esta región se encuentra la planta potabilizadora Mérida I que provee agua potable a los habitantes de la ciudad urbana de Mérida (Ayuntamiento de Mérida, 2016).

Más específicamente, la REC se localiza en el sur del municipio de Mérida (mapa 1) y tiene una extensión territorial de 10,757 hectáreas. Su ubicación geográfica es $20^{\circ} 47^{\prime}$ y $20^{\circ} 55^{\prime}$ latitud norte y $89^{\circ} 33^{\prime}$ y $89^{\circ} 40^{\prime}$ longitud oeste. Dentro de su extensión se encuentran las siguientes localidades comisarias Molas, Dzununcán y diez subcomisarias Xmatkuil, Tahdzibichén, San Pedro Chimay, Hunxectamán, San Ignacio Tesip, Dzoyaxché, Santa Cruz Palomeque y otras colonias pertenecientes a la ciudad de Mérida. 


\section{Mapa 1. Ubicación de la Reserva Ecológica Cuxtal}
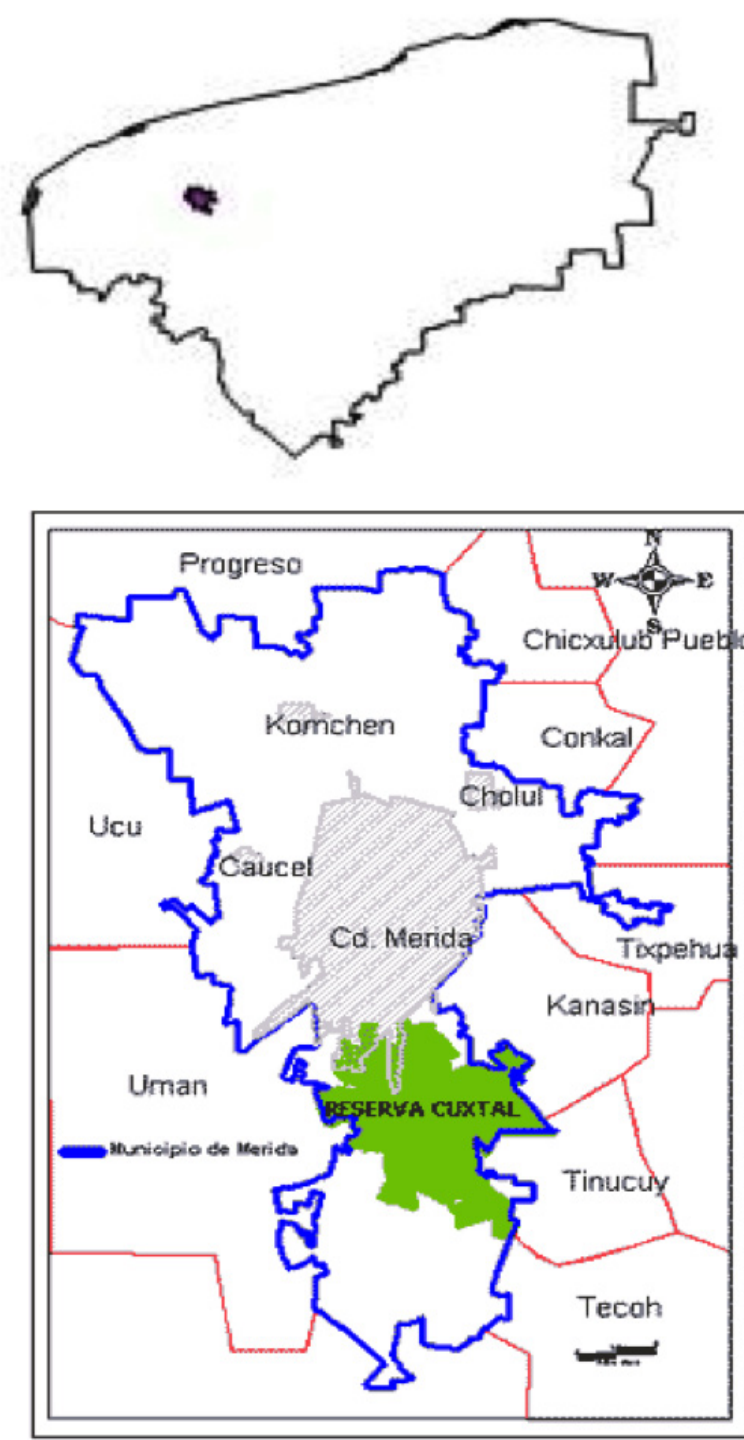

Fuente: Reserva Ecológica Cuxtal (2016)

Con relación de las características de la población ocupada (PO) entre los tres sectores económicos se tiene que para el sector primario, la mayor parte proporcional de su PO se localiza en la localidad de Hunxectamán. Para el sector secundario, la mayor proporción de la PO dedicada a este sector es Tahdzibichén representando el 56\% comparado con otras localidades. Ahora para la mayor proporción del PO dentro del sector terciario es la localidad de Santa Cruz Palomeque con 77\%. 


\section{Gráfica 1. Ingreso por trabajador semanal (YPTS) por localidades en RECW}

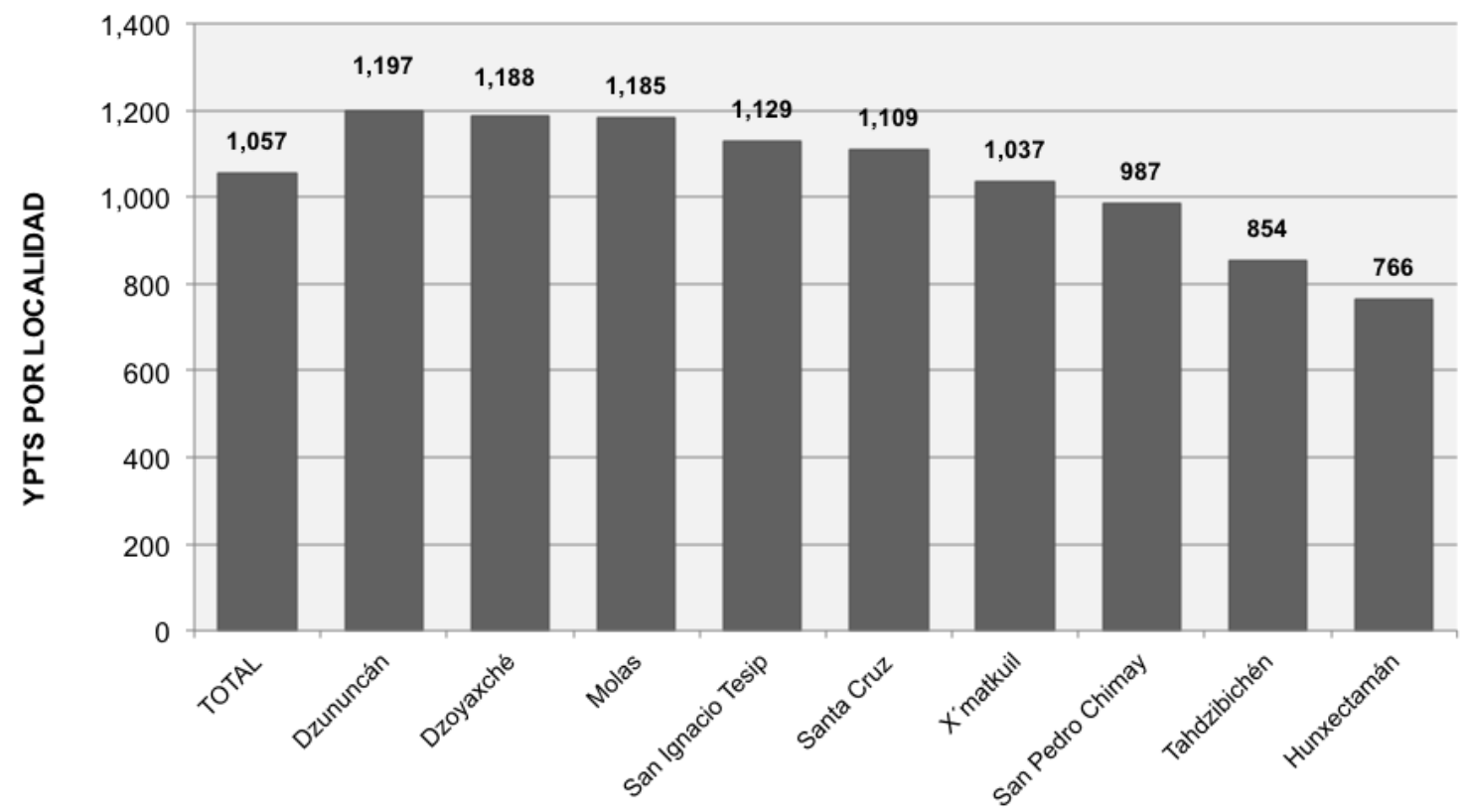

Fuente: Datos generados de encuesta socioeconómica aplicada (2014)

En cuanto al nivel de ingresos recibidos por la PO se encuentra que la mayor parte de los trabajadores dentro de la REC rondan alrededor de cuatro salarios mínimos siendo el ingreso promedio semanal equivalente a $\$ 1,057.00$ pesos semanales lo que equivale a $\$ 151$ diarios. Si se toma en cuenta el valor de $\$ 68.28$ como salario mínimo para esta zona, entonces significa que el trabajador promedio de la REC gana menos de dos salarios mínimos (SCHP, 2015). Otra parte, el 7\% de la PO obtiene entre \$273.13 y $\$ 409.68$ diarios, $1 \%$ de la $\mathrm{PO}$ entre $\$ 409.69$ y $\$ 546.24$ mientras que solo el $2 \%$ de la PO gana más de $\$ 574.42$ diarios. La representación de los ingresos por trabajador a la semana se muestra en la gráfica 1 en la cual se muestra que es en Dzununcán donde se percibe el mayor monto.

\section{Resultados}

\subsection{Crecimiento población en la REC}

La población dentro del REC ha tenido un comportamiento creciente ya que se observa un incremento de ésta. Según INEGI (2010) hay nueve localidades del municipio y diez colonias de Mérida involucradas. En las localidades hay 8,112 habitantes y 15,925 en las colonias, este último representa el 66\% de la población y se ubican a lo largo de la carretera Mérida-Xmatkuil en la parte norte de la REC. 
Gráfica 2. Población de la REC* (1950-2010) y su proyección hasta el año 2050

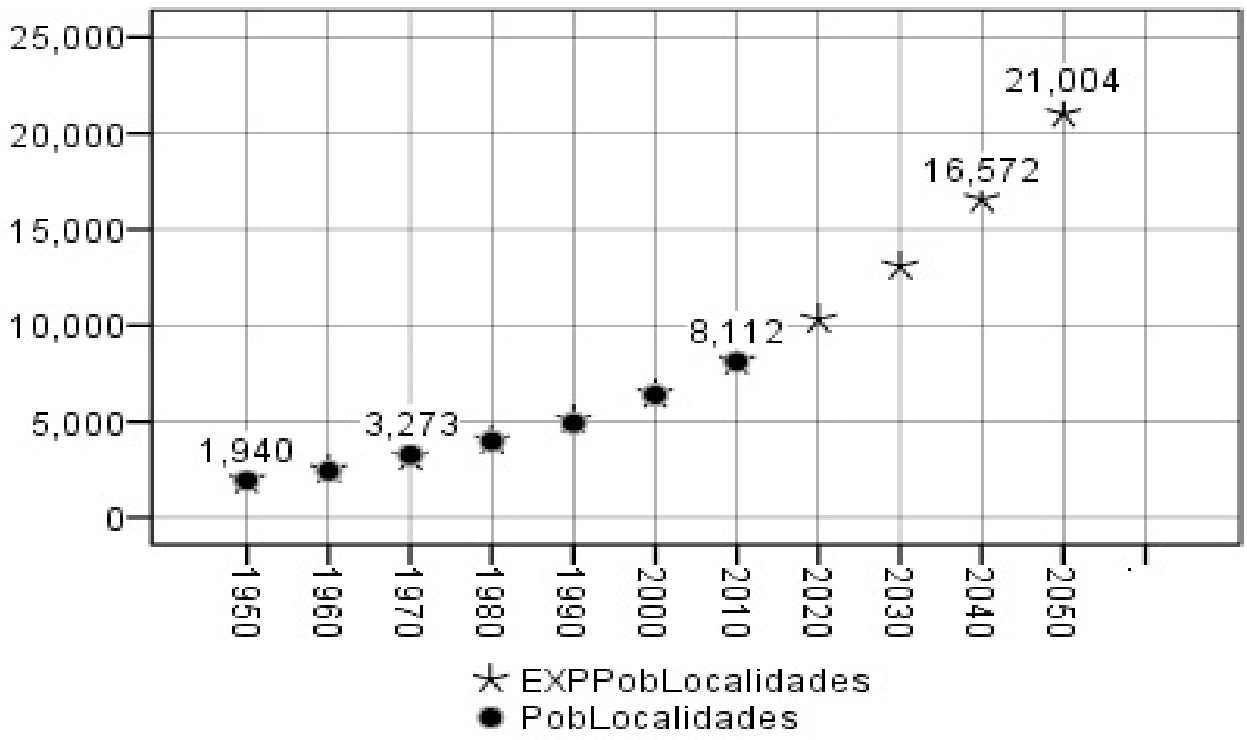

Fuente: Celis (2015). ${ }^{\star}$ Incluye solo comisarías y subcomisarias.

Con relación al uso del suelo, según el Ayuntamiento de Mérida (2016), la distribución sería de 1,564 hectáreas para uso humano, 379 hectáreas para uso urbano y 1,185 hectáreas para realizar actividades agropecuarias; de las 10,757 hectáreas totales de la REC, es decir, el 29\% ya utilizado. Es fácil ver que no hay uso sustentable sobre el suelo y la contaminación del agua era habitual por parte de las viviendas de la ciudad de Mérida aunque en la zona se encuentra la planta potabilizadora de agua y se reporta la buena calidad de agua (Fajardo, 2012). Lo que preocupa es la expansión poblacional ya que provoca mayores desechos humanos y que puede caer en el manto freático contaminando el agua de la ciudad de Mérida. Para conocer la presión que tendría en el futuro de la REC, con solo hacer un pronóstico hacia el año 2050 se puede observar la presión que tendría la población. Esta estimación se utiliza como insumo la población histórica a partir del año 1950 hasta 2010. En base a esta serie histórica se proyecta el comportamiento de la población de 2010 a 2050. Los resultados muestran un incremento de 8,112 a 16,572 en 2040 (duplicación) (gráfica 2).

Esa misma prospección de la gráfica 2 se puede realizar pero tomando en cuenta a las comisarías, subcomisarias y las colonias involucradas en la REC lo que incrementa la población de la serie histórica. Se aproxima a un comportamiento exponencial. Los resultados muestran que la población se incrementará en casi 500\% tomando como base el año 2010 hasta el año 2050, mientras que para el año 2030 se esperaría superar la duplicación de la población (gráfica 3). 
Gráfica 3. Población de la $\mathrm{REC}^{\star}$ y su proyección hasta el año 2060

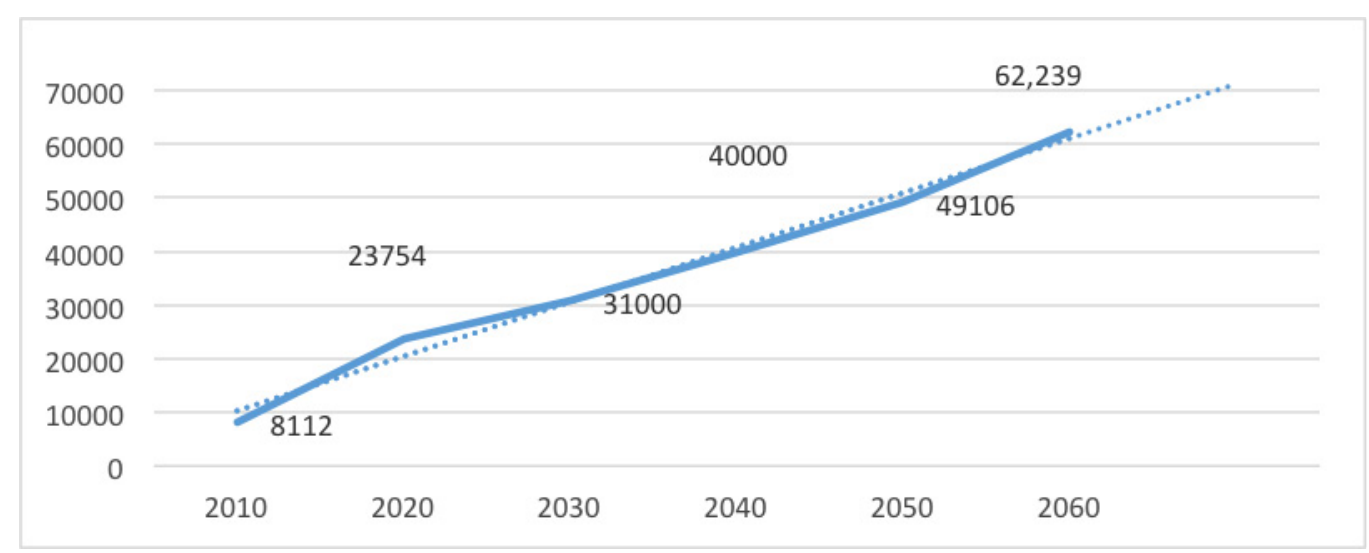

Fuente: Elaboración propia. ${ }^{\star}$ Incluye comisarías, subcomisarias y colonias

Otro cálculo sobre la población y su efecto en la REC lo representa el gráfico 3. Este muestra como el crecimiento de la población reduce las hectáreas pertenecientes a la REC. Se puede ver que para el año 2015 se ha "invadido" el 10\% de la REC mientras que alrededor del año 2085 manteniendo las cosas sin cambio alguno, se habrá ocupado casi el 50\%. Ya para el año 2115 se habrá practicamente invadido toda el área perteneciente a esta reserva.

Es importante mencionar que la superficie asignada a la REC es de 10,757 hectáreas, por tanto, en términos de hectáreas equivale a que en el año 2020 se habrá invadido 1,198 has, en el año 2050 unos 2,440 has, mientras que si mantiene esta tendencia para el año 2090 ya se habrá cubierto 6,295 has y ya para el año 2115 ya se habrá invadido el total del área asignada ${ }^{6}$.

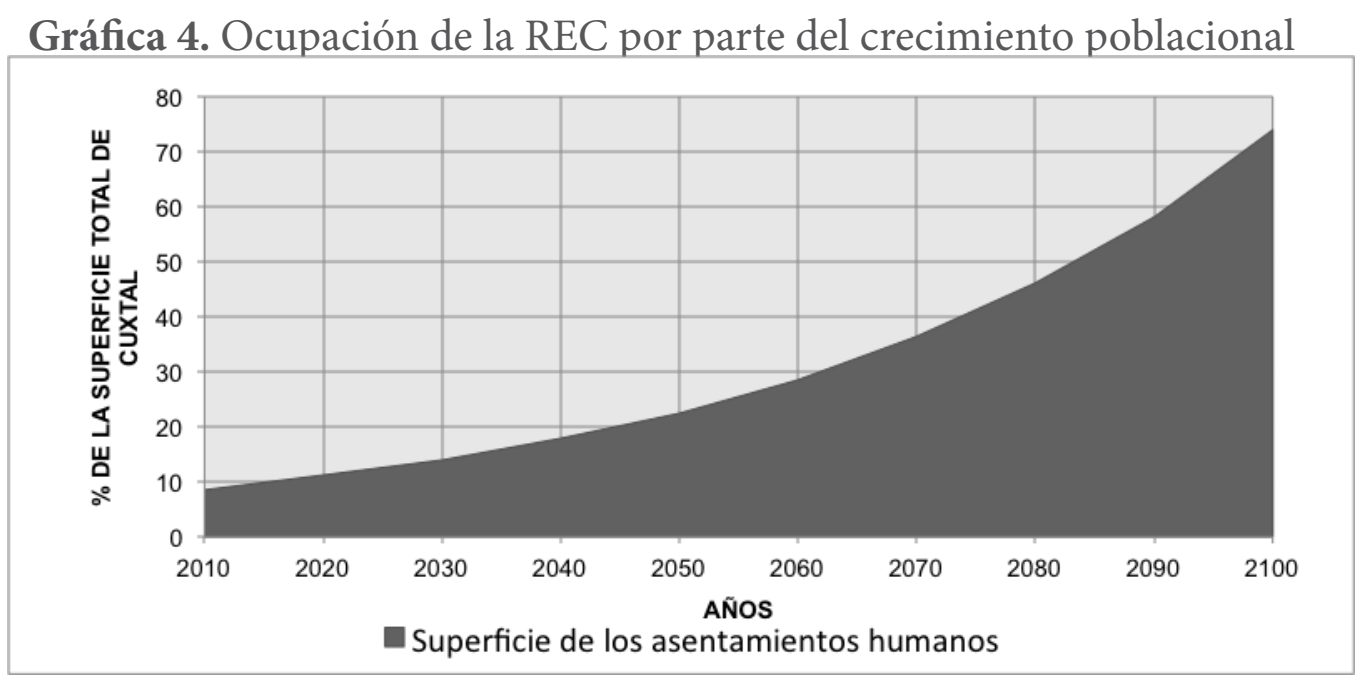

Fuente: Celis (2015).

6- Para el año 2020 la densidad poblacional por kilómetro cuadrado será de 11.98, para el año 2050 será de 24.4 y para el año 2090 de 62.96. 
Los resultados de la gráfica 4 parecieran ser sobreestimados, no obstante, estos son posibles considerando que el aumento poblacional de las colonias de Mérida tienen un crecimiento exponencial. Lo importante de este análisis independientemente de los datos, es que esta población dentro de la REC ocasionaría mayor contaminación del suelo y agua con todos los residuos contaminantes que pudiera generar si no existiera un control ambiental.

\subsection{Marginación en la REC}

La condición de marginación dentro de la REC es también un problema como en varias sociedades al grado que para las localidades pertenecientes a la reserva presentan un alto nivel de marginación (CONAPO, 2015). Aunque en el municipio, Mérida presenta bajo nivel de marginación, no es así cuando se presenta a nivel localidades. De hecho, alrededor del 60\% de las comunidades que pertenecen a la REC presentan un alto grado de marginación lo que corresponde a nueve localidades.

Si se toma en cuenta las colonias de Mérida, el grado de marginación se eleva al 65\% ya que en la zona sur de Mérida (o zona de la REC) es donde existen más carencias de servicios públicos y bajos salarios recibidos. Con ese porcentaje, la cantidad de población involucrada y que presenta este problema es alrededor de 24,036 personas. En términos generales, dentro de la REC existen 229 viviendas sin excusado, 103 viviendas sin agua entubada y 35 viviendas sin energía eléctrica (cuadro 2).

Cuadro 2. Características de las viviendas en las localidades de la REC

\begin{tabular}{|c|c|c|c|c|c|c|}
\hline Localidad & $\begin{array}{l}\text { Viviendas } \\
\text { habitadas }\end{array}$ & $\begin{array}{l}\text { Viviendas sin } \\
\text { excusado }\end{array}$ & $\begin{array}{c}\text { Viviendas } \\
\text { sin electricidad }\end{array}$ & $\begin{array}{c}\text { Viviendas } \\
\text { sin agua } \\
\text { entubada }\end{array}$ & $\begin{array}{l}\text { Población } \\
\text { total }\end{array}$ & $\begin{array}{c}\text { Grado } \\
\text { margina- } \\
\text { ción }\end{array}$ \\
\hline 1.Molas & 504 & 24 & 2 & 4 & 2,014 & Alto \\
\hline 2.Dzununcán & 397 & 10 & 1 & 3 & 1,802 & Alto \\
\hline $\begin{array}{l}\text { 3.San Pedro Chi- } \\
\text { may }\end{array}$ & 276 & 34 & 5 & 13 & 1,241 & Alto \\
\hline $\begin{array}{l}\text { 4.Santa Cruz } \\
\text { Palomeque }\end{array}$ & 190 & 20 & 3 & 5 & 835 & Alto \\
\hline 5.Tahdzibichén & 164 & 13 & 1 & 5 & 724 & Alto \\
\hline 6.Xmatkuil & 122 & 11 & 5 & 12 & 526 & Alto \\
\hline 7.Dzoyaxché & 108 & 29 & 4 & 21 & 454 & Alto \\
\hline 8.San Ignacio Tesip & 84 & 23 & 0 & 0 & 359 & Alto \\
\hline 9.Hunxectamán & 38 & 37 & 0 & 16 & 156 & Alto \\
\hline Subtotal REC & 1,883 & 201 & 21 & 79 & 8,111 & Alto \\
\hline $\begin{array}{l}\text { Colonias sur } \\
\text { de Mérida }\end{array}$ & 4,198 & 28 & 14 & 24 & 15,925 & MA \\
\hline Total & 6,081 & 229 & 35 & 103 & 24,036 & Alto \\
\hline
\end{tabular}

Fuente: Celis (2015). MA= Muy alto 


\subsection{Percepción social de la REC}

Los resultados obtenidos de los cuestionarios aplicados a la población en la REC abordan aspectos sobre la importancia de la sociedad hacia el uso del suelo así como del ambiente social que se vive. De igual modo se pregunta sobre si la población está consiente que vive dentro de un área protegida.

Con relación al uso del suelo, se tiene que a pesar de que mucha fuerza laboral se desplaza a la ciudad capital del estado (Mérida), la dependencia con los recursos naturales como el suelo y leña aún es importante. Solamente el $10 \%$ de las familias ${ }^{7}$ realiza milpa para la siembra de maíz y frijol lo que sirve de ayuda para el gasto familiar, aunque indican que el rendimiento por hectárea ha disminuido comparado al año 2004 debido a la falta de lluvias pues no cuentan con sistemas de riego.

Otras familias (27\%) practican actividades productivas dentro de su solar, entendido como el área alrededor de la vivienda habitada que destinan para producir algunas hortalizas y crianza de algunos animales de traspatio (pollos, pavos, cerdos, etc.). Los productos obtenidos sirven de igual modo como una ayuda a sus ingresos familiares pues los utilizan para autoconsumo evitando la compra de los productos obtenidos.

Un resultado que llama la atención es la dependencia del uso de leña para ser usado como medio de combustión ya que el $85 \%$ de las familias de la REC la utiliza para cocinar sus alimentos, lo que significa que a pesar de que la población está dentro de la REC, el uso de recursos naturales es importante.

En relación a lo que piensan los dueños de tierras es interesante: $54 \%$ de ellos piensan mantener sus tierras y hacerlas producir por lo que su venta no está considerada. El 29\% de ellos piensan heredarlos a sus sucesores mientras que solamente el $15 \%$ piensan venderla a otras personas. De estos últimos, provocarían un efecto negativo para la REC pues los compradores quizás no conozcan que están comprando tierras dentro de una reserva provocando posible devastación ecológica.

Ser habitante de la REC tiene sus ventajas, pero también desafíos. Entre sus ventajas es la vida tranquila que se dan los habitantes a pesar de la cercanía de una de las urbes más importantes del estado. Sin embargo, los problemas sociales tales como drogadicción, asaltos, robos, alcoholismo, etc. están presentes.

Entre los resultados obtenidos en la realización de la encuesta se obtuvo que el 68\% de los habitantes perciben que existe alcoholismo en las calles de su localidad. Con el problema de la drogadicción, la población percibe que en su comunidad está presente en $50 \%$ de las viviendas. Casi la mitad de los encuestados piensa que existen robos y asaltos en su propia localidad. Estos problemas sociales indican que la población dentro de la REC si bien percibe una tranquilidad, éste es aparente por la presencia de esos problemas sociales en la vía pública y en algunas viviendas, sobretodo el alcoholismo en las localidades de Molas y Tahdzibichén; y de robos en San Pedro Chimay. Con relación al uso de drogas, la percepción de la población es que en Dzununcán es donde el problema es más grave.

\subsection{Desechos urbanos sólidos y límites de la REC}

Es importante darle un destino correcto a los desechos sólidos generados por los habitantes de la REC en situaciones en la que según los resultados de las encuestas, el 85\% de los entrevistados tiran los dese-

7- De las 643 familias encuestadas 
chos sin importar el lugar y otros tantos la queman; lo que provoca una contaminación del aire y suelo dentro de la misma reserva. Con esta acción surge la interrogante si los habitantes están conscientes de sus actos y si saben dónde habitan.

La encuesta indica que cerca del 60\% de los entrevistados no saben si su vivienda se encuentra dentro de la REC o en sus límites territoriales. La única comunidad que obtuvo alto porcentaje de reconocimiento que se encuentra dentro de la REC es Dzoyaxché con un 94\%, sin embargo su conocimiento es erróneo cuando indican que la REC es una hacienda ya que en ese lugar se encuentra una oficina que lucha por los intereses de la reserva; además que algunos pobladores locales e incluso no locales piensan que Cuxtal solo comprende la hacienda.

Para otras localidades dentro de la REC, el panorama no es satisfactorio ya que en Dzununcán el 73\% de los entrevistados desconoce que su localidad se encuentra dentro de la REC. Para el caso de Hunxectamán el 68\% desconoce su pertenencia, en Tadzibichén el 64\%, en Santa Cruz Palomeque el 62\% mientras que en San Ignacio Tesip son el 54\% de los entrevistados.

En otras localidades de la REC se conoce la existencia de la reserva ecológica pero no conocen sus límites. Como ejemplo se puede mencionar que el desconocimiento de los límites es del $61 \%$ de los encuestados en Dzoyaxché, el 72\% en Hunxectamán, el 75\% en Dzununcán, el 80\% en Santa Cruz Palomeque, el $82 \%$ en San Ignacio Tesip y el 86\% en Tahdzibichén.

\subsection{Casería y extracción de leña}

Debido a que la REC cuenta con habitantes que como se detalló anteriormente la mayoría no están conscientes de que habitan una reserva ecológica, se les preguntó de igual modo sobre las actividades de casería y extracción de leña dentro de la REC. Los resultados muestran que para el caso de estas actividades, la población no sabe que existe una prohibición para la práctica de estas actividades. En este sentido, el 52\% del total de entrevistados afirmó no conocer prohibiciones para esta zona. En cuatro localidades el porcentaje de desconocimiento es mayor al 50\% y dos localidades, Dzoyaxché y San Ignacio Tesip, tienen los menores porcentajes con $42 \%$ y $27 \%$ de personas que no conocen tales zonas en las cuales no está permitido la cacería y extracción de leña.

\subsection{Trabajo productivo dentro y fuera de la REC}

El trabajo productivo que se realiza dentro de reserva ecológica es diverso, pero ser peón es la peor pagada realizando actividades relacionadas con la limpieza de montes y la extracción pagada de leña. Otra actividad realizada en el campo por campesinos (milpa) es también de las más bajas. Por su parte, las mujeres se dedican a las actividades de limpieza y servicios para el hogar. 
Cuadro 3. Distribución porcentual por lugar de trabajo en las localidades de la REC

\begin{tabular}{|c|c|c|}
\hline Localidad & $\begin{array}{c}\text { Trabajadores dentro de la localidad } \\
(\%)\end{array}$ & Trabajadores fuera de la localidad (\%) \\
\hline San Pedro Chimay & 20 & 70 \\
\hline Hunxectamán & 23 & 77 \\
\hline Dzununcán & 23 & 76 \\
\hline Santa Cruz Palomeque & 24 & 71 \\
\hline Tahdzibichén & 29 & 71 \\
\hline Xmatkuil & 29 & 70 \\
\hline Dzoyaxché & 30 & 69 \\
\hline Total & 31 & 75 \\
\hline Sanacio Tesip & 25 & 76 \\
\hline
\end{tabular}

Fuente: Trabajo de campo.

Por otra parte, hay un grupo de la población trabajadora que vive dentro de la REC pero trabaja fuera de ella. Este tipo de movimiento se le conoce como "movilidad pendular" (Contreras, 2001). Este comportamiento se observa para un 75\% de los trabajadores de la REC concentrándose en San Pedro Chimay con cerca del $80 \%$ de su fuerza laboral, la cual se dedica principalmente a actividades relacionadas a la albañilería y trabajo gubernamental (cuadro 3).

En segundo lugar se ubicó Hunxectamán que por sus limitadas opciones laborales dentro de la localidad y por su población creciente hacen necesaria la búsqueda de ingreso para los hogares siendo al exterior de ésta donde lo encuentran, realizando actividades propias de los obreros y empleados. De igual modo, en Dzununcán, según los resultados de la encuesta, la mayoría de su población sale de su comunidad para dedicarse en actividades de la construcción o en la prestación de servicios. Por su parte, en la localidad de Santa Cruz Palomeque con el 76\% de su población que trabaja fuera, la mayoría en empresas cuya actividad principal son los servicios de limpieza así como labores domésticas en hogares principalmente de Mérida.

En general, las actividades productivas que se realizan fuera de la REC son trabajos propias de los obreros, trabajadores de gobierno, trabajadores por cuenta propia, artesanos y servicios profesionales; sin embargo, los trabajadores regresan a sus localidades diariamente debido a la relativa cercanía entre sus centros de trabajo y sus hogares. 


\section{Gráfica 5. Diagrama de caja: Ingreso semanal (ysemanal) y de movilidad laboral de la Población ocupada en la Reserva Ecológica Cuxtal}

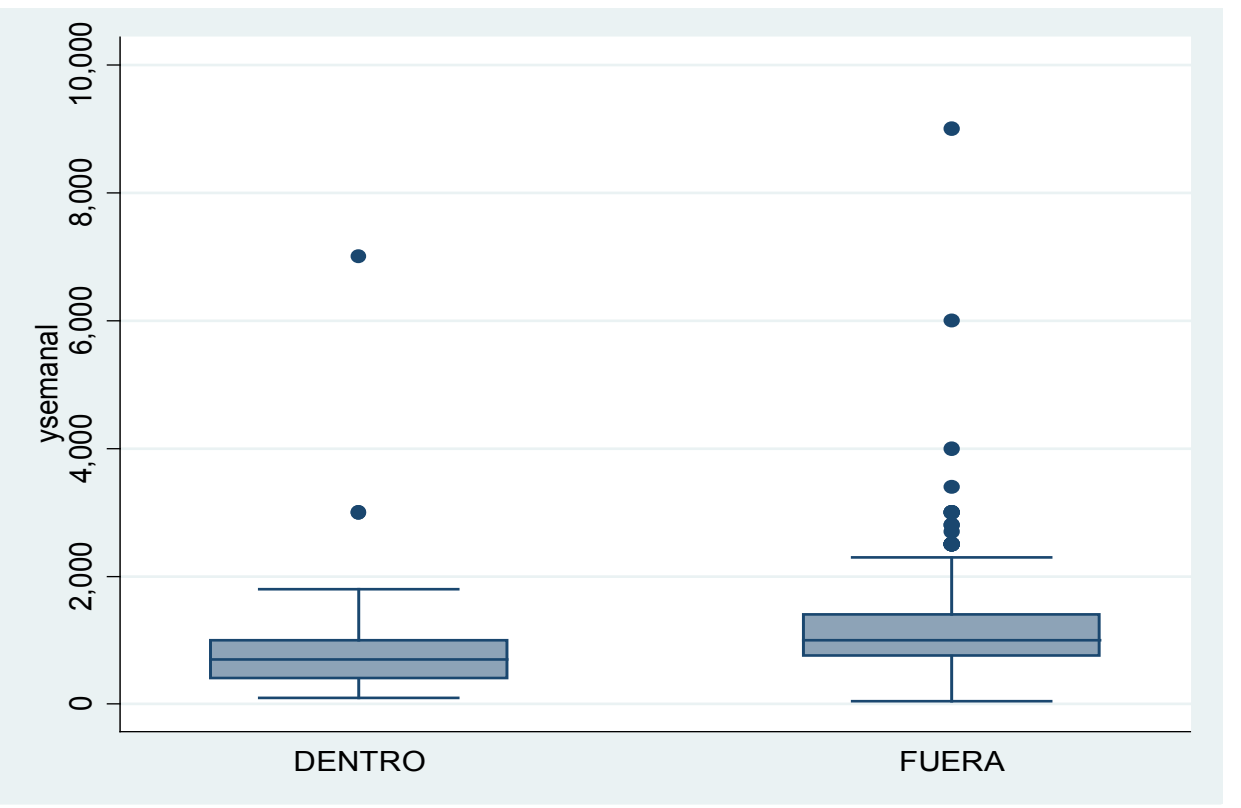

Fuente: Elaboración propia con datos de la encuesta.

De igual modo, según los resultados de las encuestas aplicadas durante 2014, se puede establecer que los mejores pagados son los obreros como albañiles (industria de la construcción) con \$1,236.00 semanales, al igual que las actividades relacionadas con los servicios profesionales, la prestación de servicios públicos así como los servicios técnicos y de mantenimiento (\$1,227.00 semanal). Por el lado contrario, entre los peores pagados se encuentran las actividades relacionadas con las actividades agrícolas tales como la limpieza del monte para hacer la milpa y la venta de leña, actividades que afectan a la conservación de la reserva ecológica (gráfica 5).

Como se observa, estas actividades deberían considerarse que alivian la presión sobre la REC; sin embargo es el crecimiento poblacional la que ejerce la mayor degradación de los recursos naturales disponibles de la reserva ecológica. Por tanto, que una gran proporción de la población esté trabajando fuera de la reserva no se toma como un factor determinante para una menor afectación sobre la REC, al contrario; favorece a que los espacios o terrenos sean vendidos a personas fuera de las localidades lo que aumenta el desconocimiento de la existencia de la reserva ecológica.

De hecho, este comportamiento se puede agravar por los niveles salariales que pueden incrementarse fuera de la REC pues la probabilidad de que un habitante se vaya fuera de la REC por trabajo ante un incremento salarial es positivo, es decir; es más probable que un habitante se desplace a trabajar fuera de la reserva si hay incrementos de salarios en otras zonas o ciudades. Este argumento se demuestra por medio de usar el modelo probit binomial en STATA $^{\circ}$ en la cual se denota 1 al trabajador que sale a trabajar de la REC y 0 lo contrario. Para fines prácticos, se usa "mov" como variable dependiente (si trabaja dentro o fuera de la REC) y “ysemanal” para denotar el ingreso semanal.

$\mathrm{Al}$ correr el modelo (regresión probit) con constante se obtiene el resultado en la cual la variable "ysemanal" es estadísticamente significativa dado el valor de z y el valor estadístico WALD (17.40). De igual 
modo, es para el valor de LR y el porcentaje de predicciones correctas (correctly classified) que en este caso es del $76.53 \%$ (cuadro 4 ).

Cuadro 4. Resultados del modelo probit

\begin{tabular}{|c|c|c|c|c|}
\hline Variable & Coeficiente & Error estándar & $\mathbf{Z}$ & $P>|Z|$ \\
\hline ysemanal & 0.00044 & 0.0001055 & 4.17 & 0.000 \\
\hline C & 0.2918473 & 0.1191476 & 2.45 & 0.014 \\
\hline \multicolumn{5}{|c|}{ Efecto marginal de ysemanal sobre la movilidad } \\
\hline Variable & $\mathrm{dy} / \mathrm{dx}$ & Error estandar & $\mathbf{Z}$ & $P>|Z|$ \\
\hline ysemanal & 0.0001319 & 0.00003 & 4.23 & 0.000 \\
\hline
\end{tabular}

Fuente: Elaboracion propia con resultados de STATA $\quad n=463$ trabajadores, $L R=19.22 \quad{ }^{2}[($ mov $)$ ysemanal $=0]=$ 17.40; Pseudo R2 $=0.0373$; Correctly classified $=76.53 \%$

Para conocer la probabilidad de decisión de movilidad pendular por cada incremento en un valor de un peso mexicano se obtiene el efecto marginal del "ysemanal". El resultado que se obtiene muestra un signo positivo de 0.0001319 (dy/dx) lo que se puede interpretar como "por cada incremento en un peso mexicano, la probabilidad de movilidad aumenta en 0.01319\%”.

\section{Conclusiones y recomendaciones}

Al concluir este estudio sobre la situación de la Reserva Ecológica Cuxtal (REC) se puede argumentar lo siguiente:

Es lamentable que el "pulmón” de una ciudad importante del sureste mexicano, Mérida, esté perdiendo una reserva ecológica que se estableció con bases claras e importantes beneficios ambientales. La mayoría de la población que habita en las localidades dentro de la REC desconocen que sus comunidades se ubican al interior de la reserva; de hecho, en muchos casos no conocen los límites de Cuxtal, lo que hace evidente la falta de interés real de las diferentes administraciones de gobierno por implementar acciones y destinar recursos encaminados a inculcar la valoración del área así como la concientización ambiental. No se puede entender como las personas piensan que la REC es solo la localidad de Dzoyaxché y su hacienda. 
La condición anterior se agrava en cuanto a la presión demográfica que existe en la parte norte de la REC, esto es la expansión urbana de la ciudad que abarca casi 562 hectáreas que representa el 10\% de la superficie total de la reserva ecológica. De hecho, el pronóstico de estos asentamientos humanos es que se puedan duplicar en el año 2045 cubriendo el 20\% de la reserva.

- Con relación al lugar de trabajo de la población ocupada se concluyó que gran porcentaje de esta se desplaza a la ciudad de Mérida (capital del estado) ya que en las localidades ubicadas dentro de la reserva no hay opciones de trabajo bien remunerados y los que permanecen en las comunidades reciben un nivel bajo de salarios comparado con los que salen a trabajar. Se muestra según los resultados de la encuesta realizada en el año 2014 que entre los trabajos que se practican internamente son diversos pero sobresalen la limpieza de terrenos, servicios de mantenimiento, albañiles y otras actividades de servicios.

El hecho de ser habitantes de la reserva y al mismo tiempo disponer de una ocupación principal fuera de su localidad, puede dar pie a suponer que no existen conflictos entre pobladores y las funciones por las que fue decretada la reserva. Sin embargo se pudo detectar que si existen conflictos entre ejidatarios (dueños de tierras) y pobladores por diferencias de opinión e intereses, ya que algunos consideran que están limitadas sus opciones por las restricciones consustanciales al funcionamiento de la reserva.

- El análisis PROBIT muestra la probabilidad que ante un aumento de los ingresos semanales en actividades productivas realizadas fuera de la REC se puede producir una mayor movilidad laboral. Los resultados mostraron que existe una relación positiva entre esas variables e indican por ejemplo que si les ofrecieran $\$ 600.00$ a la semana existirá un $70 \%$ de probabilidad que acepten el trabajo fuera de la REC, mientras que si les ofrecieran $\$ 2,100.00$ pesos mexicanos la probabilidad aumenta en $90 \%$. Estos resultados muestran la dependencia laboral con la capital del estado pero que puede tener un impacto sobre el uso del suelo en la reserva. Sin embargo, su impacto sería más significativo por el incremento poblacional que se espera que tenga el área geográfica que abarca la REC.

Ante tales resultados, se recomienda realizar campañas de educación ambiental y concientización sobre la existencia de la REC y no deberían encaminarse únicamente hacia Dzoyaxché y su hacienda (que muchos consideran como la REC) sino a otras localidades que integran la reserva. Por tal motivo, esas campañas deberían de realizarse para explicar el motivo de la creación de la reserva así como las ventajas que trae a las comunidades involucradas y sobre todo que la REC provee agua potable a la capital del estado y que la riqueza de flora y fauna aun vivientes tienen un valor invaluable para la sociedad.

Con relación a las demás comisarías y subcomisarias dentro de la reserva, debería de promocionarse como destino ecoturístico para los habitantes del municipio de Mérida pues la mayoría de los habitantes de esta ciudad no conocen la existencia de la REC; por tanto, no valoran su importancia para la ciudad capital. La creación de un museo ecológico así como paradores ecoturísticos en las comunidades de la reserva puede ser de beneficio para los pobladores en cuanto les proveerían ingresos por los servicios que puedan prestar al visitante mientras cuidan la reserva. De este modo, los dueños de las tierras dentro de la reserva tendrían una opción más sustentable para aprovechar sus tierras mientras que por otro lado se participa y contribuye al cuidado del medio ambiente ubicado en la reserva ecológica. 


\section{Referencias}

Ayuntamiento de Mérida. 2016. Mérida.gob.mx. http://www.merida.gob.mx/ecomerida/cuxtal001.htm (8 de diciembre de 2014).

Celis, Henry. 2015. Análisis poblacional y laboral de los habitantes de la Reserva Ecológica Cuxtal, 2014: Una propuesta desde la sustentabilidad. Tesis de licenciatura en economía. Universidad Autónoma de Yucatán.

CONANP. 2016. Áreas Naturales Protegidas. Comisión Nacional de Áreas Naturales protegidas. http:// www.conanp.gob.mx/regionales/ (15 de enero de 2016).

CONAPO. 2015. Indice de marginación por localidad, 2010. Consejo Nacional de Población. http:// www.conapo.gob.mx/es/CONAPO/Indice_de_Marginacion_por_Localidad_2010 (27 de febrero de 2015)

Contreras, Camilo. 2001. Geografía del mercado de trabajo en la Cuenca Carbonífera de Coahuila. Revista Frontera Norte, 13 (2): 87-118.

Diario Oficial del Gobierno del Estado de Yucatán. 2004. Diario Oficial. http://www.conacyt.mx/cibiogem/images/cibiogem/sistema_nacional/documentos/ANPL/Yuc/9-prog-manejo-cuxtal.pdf (10 de febrero de 2016).

Díaz, Reynol y Susana Escárcega. 2009. Desarrollo sustentable: oportunidad para la vida. México: Editorial Mc Graw Hill Educación http://siar.regionpuno.gob.pe/public/docs/1106.pdf (15 de enero de 2016). Fajardo, José. 2012. Recursos en disputa: la construcción social de la Reserva Ecológica Cuxtal en Dzoyaxché, Yucatán. Mérida; México. Universidad Autónoma de Yucatán.

http://www.antropologia.uady.mx/titulados/egresado.php?id=293 (10 de febrero de 2016).

García-Gil, Gerardo y Javier Escalante. 2013. Ordenamiento Territorial del estado de Yucatán: Visión 2030. Mérida, Yucatán. Editorial Universidad Autónoma de Yucatán.

García-Gil, Gerardo; Yolanda Oliva-Peña y Rafael Ortiz-Pech. 2012. Distribución espacial de la marginación urbana en la ciudad de Mérida. Investigaciones Geográficas, Boletín. Instituto de Geografía, UNAM. 77: 67-81. DOI: http://dx.doi.org/10.14350/rig.31021.

Gonzáles, Héctor; Patricia Cortés, Luis Íñiguez y Alfredo Ortega. 2014. Las áreas naturales protegidas de México. Investigación y Ciencia de la Universidad Autónoma de Aguascalientes, 60: 7-15.

Hardín, Garrett. 1968. La tragedia de los comunes. Science Review (162): 1243-1248. https://www.uam.es/ personal_pdi/ciencias/jonate/Eco_Rec/Intro/La_tragedia_de_los_comunes.pdf (20 de enero de 2016).

INEGI. 2010. ITER 2010. Instituto Nacional de Estadística y Geografía. http://www.inegi.org.mx/sistemas/consulta_resultados/iter2010.aspx (27 de febrero de 2015).

Iracheta, Alfonso y Jorge Bolio. 2012. Mérida Metropolitana, Propuesta integral de desarrollo. Mérida: Fundación Plan Estratégico de Yucatán A. C.

Lugo, José y Lizbeth Tzuc. 2010. Conurbación en el municipio de Mérida: su impacto en la economía rural y el ambiente. En FMAM, PNUD, SGP, CICY, CONABIO, C. B. Mesoamericano, y otros, Biodiversidad y Desarrollo Humano en Yucatán: 77-81. Mérida.

Mendenhall, William., Lyman Ott y Richard Scheaffer. 1987. Elementos de muestreo. México: Grupo Editorial Iberoamérica. 
Merchant, Marco. 2011. El estilo de desarrollo que hace inviable el "desarrollo sustentable" de México. Paradigma económico, 3 (2): 33-60

Miguel, Andrés, Julio Torres, Pedro Maldonado y Juan Robles. 2011. Las desigualdades regionales del desarrollo sustentable en México, 2000-2005. Revista Región y Sociedad, 23 (51): 101-122.

Ostrom, Elinor. 2000. El gobierno de los bienes comunes. La evolución de las instituciones de acción colectiva. (Corina de Iturbide Calvo y Adriana Sandoval, traductores), México: Universidad Nacional Autónoma de México, Centro Regional de Investigaciones Multidisciplinarias y Fondo de Cultura Económica. Reserva Ecológica Cuxtal. 2016. Ubicación de la Resrva Ecológica Cuxtal. https://infogr.am/ReservaEcologica-de-Cuxtal (10 de mayo de 2016).

Rodríguez, Luis. 2010. El gobierno de los bienes comunes, la evolución de las instituciones de acción colectiva. Revista Pueblos y Frontera Digital, 6 (10): 363-375.

Ruiz, Héctor y José Arrellano. 2016. Instrumentos y estrategias. Áreas Naturales Protegidas. http://www. cicy.mx/Documentos/CICY/Sitios/Biodiversidad/pdfs/Cap8/05\%20Areas\%20naturales\%20protegidas. pdf (10 de febrero de 2016).

SEDUMA. 2016. Bitácora ambiental del Programa de Ordenamiento Ecológico Territorial del Estado de Yucatán. http://www.bitacoraordenamiento.yucatan.gob.mx/marco/index.php (5 de mayo de 2016).

SHCP. 2015. Salarios mínimos 2015. Secretaría de Hacienda y Crédito Público. http://www.sat.gob.mx/ informacion_fiscal/tablas_indicadores/Paginas/salarios_minimos.aspx ( 28 de mayo de 2015).

\section{Agradecimientos}

Los autores quieren agradecer al financiamiento otorgado por el Ayuntamiento de Mérida Administración 2012-2015 para la realización de este proyecto de investigación con la colaboración de dos cuerpos académicos de Desarrollo Económico Sustentable (CADES) y de Diversidad de los Recursos Florísticos de Mesoamérica, ambos de la Universidad Autónoma de Yucatán (UADY). 